
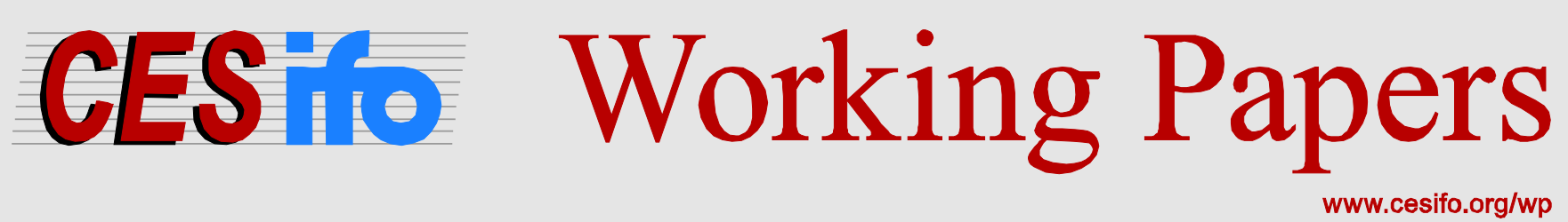

\title{
Information Aggregation in Democratic Mechanisms
}

\author{
Volker Britz \\ Hans Gersbach
}

CESIFO WORKING PAPER NO. 5815

CATEgORy 2: Public CHOICE

MARCH 2016

An electronic version of the paper may be downloaded

- from the SSRN website:

- from the RePEc website:

- from the CESifo website:

WwW.SSRN.com

www.RePEc.org

www.CESifo-group.org/wp 


\title{
Information Aggregation in Democratic Mechanisms
}

\begin{abstract}
We examine whether and how democratic procedures can achieve socially desirable public good provision in the presence of profound uncertainty about the benefits of public goods, i.e., when citizens are able to identify the distribution of benefits only if they aggregate their private information. Some members of the society, however, are harmed by socially desirable policies and aim at manipulating information aggregation by misrepresenting their private information. We show that information can be aggregated and a socially desirable policy can be implemented under a new class of democratic mechanisms involving a sample group. These mechanisms reflect the principles of liberal democracy, are procedurally efficient, and involve a conditional tax privilege of sample group members. This tax treatment motivates sample group members to reveal their private information truthfully before voting takes place. Depending on the distance between two feasible public good levels, the optimal mechanism involves either one or two voting rounds. We show that procedural efficiency cannot be achieved by communication among all citizens prior to voting. Finally, we outline several applications of the mechanism.
\end{abstract}

JEL-Codes: D620, D720, H400.

Keywords: democratic mechanisms, polling, sampling, public goods, voting, information aggregation.

Volker Britz

Center of Economic Research

ETH Zurich (CER-ETH)

Zuerichbergstrasse 18

Switzerland - 8092 Zurich

vbritz@ethz.ch
Hans Gersbach

Center of Economic Research

ETH Zurich (CER-ETH)

Zuerichbergstrasse 18

Switzerland - 8092 Zurich

hgersbach@ethz.ch

This version: January 22, 2016

The research presented in this paper has benefited from discussions with Carlos Alos-Ferrer, Clive Bell, Patrick Bolton, Tilman Börgers, Martin Hellwig, César Martinelli, and Francesco Squintani, and from the comments of conference audiences at SING10 in Krakow and the 2014 EEA meeting in Mannheim. Seminar participants at universities in Davis, Heidelberg, Irvine, Munich, San Diego, and Zurich have provided valuable comments and suggestions on democratic mechanisms. A precursor of the current manuscript has appeared as a working paper by Britz and Gersbach (2014) under the title "Experimentation in Democratic Mechanisms." 


\section{Introduction}

The ability of democratic decision-making procedures to achieve socially optimal outcomes is at the core of a long-standing and complex debate with many unresolved issues. One challenging question, in particular, is whether democratic procedures can resolve profound uncertainty. We use the term "profound uncertainty" to refer to situations in which individual costs and benefits from public policies are privately observed realizations from a probability distribution which depends on an unknown state variable. That is, profound uncertainty involves ambiguity on top of uncertainty.

The problem we consider in this paper is that of a society which has to decide collectively on the quantity of a public good which is financed by uniform taxation. There is a finite number of feasible public good levels. An individual citizen's benefit from the public good depends on the citizen's privately observed type. These types are drawn from a probability distribution, which itself depends on the state of the world. The state of the world is not known to any citizen, but all citizens have a common prior belief about it. If the citizens aggregate their private information, they can learn the state of the world. In each state of the world, there is a Condorcet winner, that is, there is one feasible public good level which is preferred by the majority to any other feasible public good level. However, the Condorcet winner differs across states. In this setup, the implementation of the socially desirable outcome hinges crucially on the aggregation of citizens' private information. Given sufficiently diverse preferences of the different citizens, it is clear that some citizens can have incentives to strategically misrepresent their private information.

We are interested in finding a collective decision-making procedure which motivates citizens to reveal private information truthfully. Specifically, we are interested in "democratic mechanisms," that is, decision-making procedures which respect some important democratic principles. Contrary to a standard mechanism design problem, the typical citizen can only send binary messages, that is, say "Yes" or "No." Moreover, the questions or proposals to which the typical citizen answers by a binary message are not made by an outside designer or planner but are put to a vote by citizens who are themselves members of the society. One more feature of a democratic mechanism is that the status quo can only be replaced with some feasible alternative by a majority vote. However, once the majority has come to a decision, all citizens must respect this decision and their financial consequences - participation constraints do not matter. Furthermore, we require a democratic mechanism to treat all citizens equally. This implies in particular that every citizen's 
vote counts equally. Finally, our aim will be to find democratic mechanisms which are efficient in the sense that they lead to the implementation of the socially desirable outcome with a limited number of voting rounds.

In the present paper, we introduce democratic mechanisms in which a small representative sample of the population communicates information about its type. In order to give the sample group members an incentive to reveal information truthfully, they are offered a conditional tax-exemption. This means that sample group members may enjoy the benefits of public good provision without bearing their share of its cost. In order to assess whether or not the privilege will be granted, the mechanism involves two rounds of voting by the population in which an original proposal is first pitted against an amendment and then against the status quo. If the original proposal prevails in both votes, the tax-exemption is granted. One main result is that this combination of prior communication by a small representative sample and the use of a conditional tax-exemption yield truthful information aggregation and consequently the implementation of the Condorcet winner in one or two voting rounds. We also provide impossibility results which indicate that the conditional tax privilege of sample group members is necessary for information aggregation.

At a more detailed level, we provide the following formal results. We start with the result that the Condorcet winner can be implemented by a stepwise elicitation mechanism with many voting rounds in which agenda-setters are motivated to propose the feasible public good levels in ascending order. We argue that this solution is not desirable because of the procedural costs. We next focus on procedural efficiency. We show that it is impossible to achieve procedural efficiency and truthful information revelation by prior communication among all citizens. Agenda-setters can exploit information revelation to engineer a voting outcome different from the Condorcet winner. Then, we introduce a new class of democratic mechanism involving prior communication by a sample group, whose random members enjoy a conditional tax privilege. We show that truthful information aggregation and procedural efficiency can be achieved in one or two popular votes, depending on the distance between two public good levels.

Our work fits into the context of a vast body of literature on optimal constitutions which has developed after the classic work of Buchanan and Tullock (1962). Aghion and Bolton (2003) have introduced incomplete social contracts and have explored how simple or qualified majority rules balance the need to overcome vested interests and respect majority preferences. Gersbach (2009) has introduced the notion of democratic mechanisms and 
has shown how increasingly sophisticated combinations of agenda rules, treatment rules, and decision rules can yield first-best allocations when each citizen faces only two possible realizations: being either a winner or a loser of a public project. The present paper extends the democratic mechanism approach to the case of profound uncertainty about valuations. Neither individual valuations nor the underlying distribution are common knowledge. In the present paper, we explore the scope of democratic mechanisms with minimal message spaces but with rich type spaces and profound uncertainty about the distribution of the types.

The democratic mechanisms discussed in the present paper relate to the polling and experimentation literatures. There is a literature on polling in which candidates for political office learn about the location of the median voter through surveys, and then decide where to position themselves on the political spectrum. Similarly to our setup, one interesting question in a polling model is whether the polled citizens find it optimal to reveal their preference truthfully. Morgan and Stocken (2008) discuss to what extent policy-makers can learn from polling. The main difference to the democratic mechanisms we propose is that polling does not distort polled citizens' preferences, whereas the tax-exemptions in our mechanisms do so, at least conditionally. Meirowitz (2005) analyzes the case of two candidates competing for political office, who attempt to elicit voter preferences through polling. A seminal contribution to the literature on strategic communication is Crawford and Sobel (1982). The limitations of polling are also discussed by Goeree and Grosser (2007) and Taylor and Yildirim (2005). Bernhardt, Duggan, and Squintani (2008) provide a recent survey of the polling literature.

Another relevant branch of literature is that of policy experimentation. In a model of experimentation, one considers a polity with a federal structure and allows one part of the society to enact a policy on its own. The remainder of the society observes this policy and its outcomes and then attempts to learn from the experience and possibly to extend the policy to the entire society. Our democratic mechanisms share with this approach the idea that one part of the society is given a special role, possibly associated with some privileges. The difference, however, is that in our model, a policy decision can only be taken for the entire society at once. At least since the seminal paper of Rose-Ackermann (1980), it is well known that the rules which govern collective decisions also determine the incentives of office-holders whether to experiment. She showed that in federal systems, free-riding reduces the incentives of candidates for office to undertake policy experiments. This line of research has been extended and deepened by Strumpf (2002), Cai and Treisman (2009), 
Volden, Ting, and Carpenter (2008), and Kollman, Miller, and Page (2000), as well as Bednar (2011). Callander and Harstad (2013) study quantity and quality of policy experimentation and characterize advantages and disadvantages of federal systems in this respect. The importance of experimentation in actual choice procedures is well established. For instance, Volden (2006) and Shipan and Volden (2006) study policy experimentation and diffusion across jurisdictions in the United States. Buera, Monge-Naranjo, and Primiceri (2011) provide a theory of policy diffusion at a global level and find empirically that learning from experience across countries is an important factor behind changes in economic policy.

The paper is organized as follows. In Section 2, we formally introduce the collective choice problem faced by our model society. Section 4 introduces the equilibrium concept needed for the analysis. In Section 3, we formalize the notion of a democratic mechanism in a list of desiderata which such mechanisms should satisfy. In Section 5, we consider a mechanism in which voting is preceded by communication. This setup resembles polling, and we argue that it is prone to manipulation in several ways. In Section 6, we then give a first implementation result. In particular, we introduce a democratic mechanism which implements the Condorcet winner under an extra restriction on the collective choice problem. In Section 7, we show that even if that restriction is dropped, a modified version of the democratic mechanism achieves implementation. Section 8 concludes.

\section{The collective choice problem}

We consider a society which consists of a continuum of risk-neutral citizens of unit mass. Citizens collectively decide how much of a public good to provide. The public good is not arbitrarily divisible, that is, the set of possible public good levels is discrete. We are interested in the decision-making process by which the society chooses from such a set of public good levels.

We will assume that the per-capita cost of providing a quantity $q \in \mathbb{R}_{+}$of the public good is given by a twice continuously differentiable, strictly increasing, and strictly convex function $^{1} c: \mathbb{R}_{+} \rightarrow \mathbb{R}_{+}$with $c(0)=0$. This implies in particular that average cost $c(q) / q$ is strictly increasing, and that $c(q) / q$ as well as $c^{\prime}(q)$ grows without bound as $q$ goes to infinity.

\footnotetext{
${ }^{1}$ Although we will eventually consider a discrete set of possible quantities, it is convenient to start by defining continuous cost and utility functions on $\mathbb{R}_{+}$.
} 
Public good provision is financed by uniform taxation so that every citizen pays $c(q)$ when $q$ is provided. Typically, one assumes that each citizen is initially endowed with $w(w>0)$ units of a private consumption good which can either be consumed or transformed into the public good. The per capita costs $c(q)$ are the utility losses due to foregone private consumption.

While the cost of public good provision is common knowledge among all citizens, there is uncertainty with regard to the benefits of the public good. Every citizen obtains a benefit from public good provision which is proportional to $q$, with the factor of proportionality given by the citizen's type. The type, in turn, is a realization of a random variable $z$ taking values in a non-empty, non-degenerate, and closed interval $Z$ in $\mathbb{R}_{++}$, and whose interior we denote by $\operatorname{int}(Z) .^{2}$ The probability distribution from which the types are drawn will be specified later. Each citizen is privately informed about his type. We will henceforth refer to the citizen of type $z$ as citizen $z$. If the public good level $q$ is provided, citizen $z$ obtains a utility of

$$
u(z, q)=z q-c(q)
$$

Moreover, we assume that one out of finitely many states of nature has been realized, and that no citizen is informed about this state of nature. We index the states of nature by $k=1, \ldots, n$ (or by $i$ and $j$ when necessary) and write $N=\{1, \ldots, n\}$. The aggregate and individual uncertainties are related as follows. When the state of nature is $k$, then the types $z$ are drawn from $Z$ by a probability distribution associated with the cumulative distribution function $F_{k}: Z \rightarrow[0,1]$. The cumulative distribution functions $\left(F_{k}\right)_{k=1, \ldots, n}$ are each twice continuously differentiable. We denote the concomitant probability density functions by $\left(f_{k}\right)_{k=1, \ldots, n}$, and their first derivatives by $\left(f_{k}^{\prime}\right)_{k=1, \ldots, n}$. We assume that $f_{k}(z)>0$ for all $k \in N$ and all $z \in Z$ and, moreover, that

$$
\frac{f_{k+1}^{\prime}(z)}{f_{k+1}(z)}>\frac{f_{k}^{\prime}(z)}{f_{k}(z)}, \forall z \in Z, \forall k \in N \backslash\{n\}
$$

The above inequality reflects a property of the family of probability distributions which is known as the monotonicity of likelihood ratios. This property has three key implications. First, the probability distribution associated with $F_{k+1}$ (strictly) first-order stochastically dominates the one associated with $F_{k}$ for every $k \in N \backslash\{n\}$, that is, $F_{k+1}(z)<F_{k}(z)$

\footnotetext{
${ }^{2}$ We denote $\mathbb{R}_{+} \backslash\{0\}$ by $\mathbb{R}_{++}$. Our main results would carry over to the case where $Z$ is only restricted to lie in $\mathbb{R}_{+}$, however, this would add a number of technical complications.
} 
for every $z \in \operatorname{int}(Z)$. In that sense, the benefits from the public good are higher in state $k+1$ than in state $k$. Second, the monotonicity of likelihood ratios implies a single-crossing property of the probability density functions, which will be crucial for our analysis. Finally, the monotonicity of likelihood ratios imposes a monotonicity property on citizens' posterior beliefs.

To be more precise, denote by $\Delta^{n}$ the unit simplex in $\mathbb{R}^{n}$ and by $\Delta_{++}^{n}$ the intersection of $\Delta^{n}$ with $\mathbb{R}_{++}^{n}$. We assume that all citizens share a common prior belief $p \in \Delta_{++}^{n}$ about the states of nature. The state space $N$ and the associated probability distributions represented by $\left(F_{k}\right)_{k=1, \ldots, n}$ are common knowledge. Upon observing his type, citizen $z$ updates the prior belief $p$ with his type $z$, thus obtaining the posterior belief $\beta(z)$ about the state of nature. According to Bayes' rule, we can write the $k^{\text {th }}$ component of $\beta(z)$ as follows:

$$
\beta_{k}(z)=\frac{f_{k}(z) p_{k}}{\sum_{j=1}^{n} f_{j}(z) p_{j}}, k=1, \ldots, n .
$$

Since $p \in \Delta_{++}^{n}$, we also have $\beta(z) \in \Delta_{++}^{n}$ for all $z \in Z$. We note that the monotonicity of likelihood ratios implies, loosely speaking, that a higher type tends to believe in higher states of nature with higher probability. We can interpret $F_{k}(z)$ as the cross-sectional distribution of $z$ in the population when the state is $k$. It is well known that this interpretation requires the application of a suitable version of the law of large numbers for a continuum of random variables. One implication is that the state of nature can be inferred by aggregating information about the realized types. We will be interested in information aggregation through binary messages.

Recall that we have defined the cost and utility functions on $q \in \mathbb{R}_{+}$. For what follows, we need to impose the following assumption.

Assumption 2.1 The type space $Z$ with its infimum inf $(Z)$, and the cost function $c$ with its first derivative $c^{\prime}$ are such that $c^{\prime}(0) \leq \inf (Z)$.

Since the image of the marginal cost function $c^{\prime}$ is $\left[c^{\prime}(0), \infty\right)$, Assumption 2.1 implies that $Z$ belongs to the image of $\mathbb{R}_{+}$under $c^{\prime} .{ }^{3}$ Together with the strict convexity of the cost function, this property implies that the preferences of each type $z \in Z$ are single-peaked, and the most preferred quantity of citizen $z$ is that $q$ which solves $c^{\prime}(q)=z$. Citizen $z$ is the median voter in state $k$ if $F_{k}(z)=1 / 2$. We define for each state $k \in N$ a quantity

\footnotetext{
${ }^{3}$ Alternatively, we could also have made the slightly stronger assumption that $c^{\prime}(q)$ tends to zero as $q \rightarrow 0$.
} 
$q_{k} \in \mathbb{R}_{+}$as the unique solution to

$$
F_{k}\left(c^{\prime}\left(q_{k}\right)\right)=1 / 2
$$

so that $q_{k}$ is the most preferred quantity of the median voter in state $k$. The definition and the assumptions regarding the cost function and the monotonicity of likelihood ratios imply that $0<q_{1}<\ldots<q_{n}$. Due to the strict convexity of the cost function, we have

$$
\begin{aligned}
& \frac{c\left(q_{k}\right)-c(q)}{q_{k}-q}<c^{\prime}\left(q_{k}\right), \forall q<q_{k}, \forall k \in N, \\
& \frac{c\left(q_{k}\right)-c(q)}{q_{k}-q}>c^{\prime}\left(q_{k}\right), \forall q>q_{k}, \forall k \in N .
\end{aligned}
$$

Due to the single-peaked preferences, these statements can be expressed in terms of the distribution functions as Ineqs. (4) and (5) below. For these expressions to be well-defined, we need to assume that $c\left(q_{1}\right) / q_{1} \in \operatorname{int}(Z)$.

$$
\begin{aligned}
& F_{k}\left(\frac{c\left(q_{k}\right)-c(q)}{q_{k}-q}\right)<1 / 2, \forall q<q_{k}, \forall k \in N, \\
& F_{k}\left(\frac{c\left(q_{k}\right)-c(q)}{q_{k}-q}\right)>1 / 2, \forall q>q_{k}, \forall k \in N .
\end{aligned}
$$

Verbally, in state $k$, a simple majority of citizens prefers $q_{k}$ over any other quantity $q \in$ $\mathbb{R}_{+} \backslash\left\{q_{k}\right\}$. Thus, $q_{k}$ is the Condorcet winner in state $k$. Moreover, for every $k \in N$, we define the quantity $\tilde{q}_{k} \in \mathbb{R}_{+}$as the unique ${ }^{4}$ solution to

$$
F_{k}\left(c\left(\tilde{q}_{k}\right) / \tilde{q}_{k}\right)=1 / 2 .
$$

In state $k$, a majority of citizens prefers any quantity $q<\tilde{q}_{k}$ to zero public good, but prefers zero public good to any quantity $q>\tilde{q}_{k}$. Given that average cost $c(q) / q$ is strictly increasing and that $c(q) / q<c^{\prime}(q)$, we have $\tilde{q}_{k} \geq q_{k}$ for every $k \in N$. For the remainder of the paper, we assume a discrete set $Q$ of possible public good levels. The set $Q$ includes the status quo denoted by $q_{0}=0$ as well as the Condorcet winners associated with the different states. Thus,

$$
Q \supset\left\{q_{0}\right\} \cup\left\{q_{1}, \ldots, q_{n}\right\}
$$

Apart from the status quo and the Condorcet winners, an arbitrary but finite number of additional quantities in the interval $\left[q_{0}, q_{n}\right]$ may belong to $Q$. It is crucial that the set of

\footnotetext{
${ }^{4}$ The uniqueness of $\tilde{q}_{k}$ follows again from the strict convexity of the cost function.
} 
feasible public good levels is discrete so that we can define

$$
\begin{aligned}
& \psi_{-}\left(q^{\prime}\right)=\max \left\{q \in Q \mid q<q^{\prime}\right\}, \text { given } q^{\prime} \in\left(0, q_{n}\right], \\
& \psi_{+}\left(q^{\prime}\right)=\min \left\{q \in Q \mid q>q^{\prime}\right\}, \text { given } q^{\prime} \in\left[0, q_{n}\right) .
\end{aligned}
$$

In the sequel, it will sometimes be relevant to consider the special case of public good problems which have the distance property defined as follows:

Definition 2.2 The public good problem $P \in \mathcal{P}$ has the distance property if $\psi_{+}\left(q_{i}\right)>\tilde{q}_{i}$ for all $i \in N$.

To sum up, we have modeled a choice situation in which all citizens know that a Condorcet winner exists, but can only identify the Condorcet winning alternative if they aggregate their private information.

The family $\left(F_{k}\right)_{k \in N}$ of cumulative distribution functions, the type space $Z$, and the cost function $c(q)$ constitute a public good problem $P$, and we denote the set of such public good problems by $\mathcal{P}$. In this paper, we are interested in mechanisms which generally implement the Condorcet winner for all public good problems $P \in \mathcal{P}$. More specifically, we are aiming at the implementation of the Condorcet winner through so-called democratic mechanisms, which we will introduce in more detail in the next section.

\section{Desiderata for democratic mechanisms}

Democratic mechanisms are meant to reflect important principles and constraints of a decision-making process in a liberal democracy. Democratic mechanisms need not satisfy individual participation constraints of the citizens. That is, decisions made through democratic mechanisms can be implemented by the coercive power of the law without the consent of every individual. For the purpose of the present paper, we think of a democratic mechanism as a collective decision-making procedure which complies with the following desiderata:

1. Minimal Message Space. Almost all citizens can send only binary messages. In particular, this implies that a citizen cannot explicitly communicate his type. This corresponds to the idea that a regular citizen can only vote "Yes" or "No" in a democratic process, rather than state his preferences in full detail. 
2. Endogenous Proposals. Agenda-setters who are a subset of zero mass of the population have a rich message space. Their role is to make proposals to which the typical citizen can react only with binary messages. Contrary to the mechanism design literature, there is no outside planner or "designer" who structures the voting process in a benevolent way so as to direct it to the socially desirable outcome.

3. Majority Voting. There is a given status quo. For some alternative to be chosen by the democratic mechanism, this alternative must defeat the given status quo in a popular vote ${ }^{5}$ under a majority rule.

4. Equal Treatment. All citizens are treated equally. In particular, agenda-setters are randomly chosen among all citizens with equal probability. Moreover, all citizens are taxed uniformly. Finally, the vote of each citizen counts equally.

5. Procedural Efficiency. A democratic mechanism should be "efficient" in the sense that it does not require a large number of popular votes. The rationale is that organizing popular votes is costly. In particular, we assume that it would be too costly to determine the state by a "brute-force" elicitation of all the $n^{2}-n$ pairwise social preferences over two alternatives. ${ }^{6}$

The purpose of this paper is to explore to what extent a mechanism can generally implement the Condorcet winner and still be "democratic" in the aforementioned sense. In Section 5 , we ask whether information can be aggregated by allowing some prior communication among the citizens before they vote, and we state two impossibility results. In Sections 6 and 7 , we combine the idea of prior communication with the instrument of a conditional tax privilege for a sample group, which eventually leads to our implementation result. We argue that the unequal treatment inherent in the tax privilege can be reconciled in several ways with the desiderata for democratic mechanisms. In particular, we argue that in a large society, the cost of the tax privilege for the sample group becomes negligible while the cost of too many voting rounds does not.

\footnotetext{
${ }^{5}$ Throughout the paper, we will use the term popular vote to describe a voting round in which all citizens (rather than just a sample) participate.

${ }^{6}$ One might wonder whether the Condorcet winner can be found by eliciting only $n$ pairwise preferences over pairs of the form $\left(q_{i}, q_{i-1}\right)$, for $i \in N$. It can be shown, however, that such a "stepwise elicitation" of preferences is always prone to manipulation, and proposals other than the Condorcet winner can be implemented. Details are available upon request.
} 


\section{Equilibrium concept}

In this section, we briefly give an overview of the equilibrium concept and some other notions which will be used repeatedly in the analysis.

In the later sections of the paper, we will define various democratic mechanisms. Combining a public good problem $P \in \mathcal{P}$ with any one of these democratic mechanisms yields a Bayesian game. In each of these Bayesian games, citizens need to make simultaneous and binary decisions when they vote on proposals or send messages. Since an individual citizen has zero mass in the continuum society, an equilibrium concept based on the absence of "unilateral deviations" would not restrict these simultaneous and binary decisions in a meaningful way. We need to work with an equilibrium concept which mimics voting behavior in a large but finite society with individuals eliminating weakly dominated strategies. $^{7}$ Therefore, we require that an equilibrium should be robust against a coordinated deviation by any group of citizens who share the same preference ranking over the discrete set of possible alternatives. Loosely speaking, whenever citizens make simultaneous and binary decisions in the game, the equilibrium concept should treat groups of citizens with the same preference ranking as if the group was a single player. ${ }^{8}$

This idea can be formalized as follows: For any type $z \in Z$, we denote by $\succcurlyeq_{z}$ the preorder over the set $Q$ such that for any $q^{\prime}, q^{\prime \prime} \in Q$, it holds that $q^{\prime} \succcurlyeq_{z} q^{\prime \prime}$ if and only $z q^{\prime}-c\left(q^{\prime}\right) \geq z q^{\prime \prime}-c\left(q^{\prime \prime}\right)$, and we refer to $\succcurlyeq_{z}$ as the preference ranking of citizen $z$ over $Q$. Let $X \subset Z$ stand for an arbitrary non-empty set of types such that $\succcurlyeq_{z^{\prime}}$ and $\succcurlyeq_{z^{\prime \prime}}$ are identical preference rankings over $Q$ for any $z^{\prime}, z^{\prime \prime} \in X$. We denote this shared preference ranking of all members of $X$ by $\succcurlyeq_{X}$.

Let $h$ be some history in the game at which citizens simultaneously make binary decisions. Consider a profile of strategies and beliefs which is a perfect Bayesian equilibrium, and let $\sigma_{h}: Z \rightarrow\{Y, N\}$ be the restriction of the equilibrium strategies to history $h$. Take a

\footnotetext{
${ }^{7}$ This is a standard procedure for the examination of voting in a continuum economy (see for instance Gersbach (2009)).

${ }^{8}$ This requirement reduces to the robustness against a deviation by an arbitrarily small group if one has sufficiently many alternatives. Another possible equilibrium concept would require the absence of a profitable deviation by a subset of players with at most arbitrarily small but strictly positive mass. However, such a concept of equilibrium would not greatly restrict the players' behavior in the game at hand. Because of the discreteness of $Q$, an arbitrarily small set of citizens with positive measure cannot affect voting outcomes and thus almost all conceivable voting patterns could be rationalized as equilibria. Such circumstances are typical of voting games and are avoided if deviations by larger groups are allowed.
} 
group $X$ with shared preference ranking $\succcurlyeq_{X}$ and a map $\hat{\sigma}_{h}: Z \rightarrow\{Y, N\}$ which satisfies $\hat{\sigma}_{h}(z)=\sigma_{h}(z)$ for all $z \in Z \backslash X$, but $\hat{\sigma}_{h}(z) \neq \sigma_{h}(z)$ for some or all $z \in X$. Then, we say that $\hat{\sigma}_{h}$ is a joint deviation by group $X$ at history $h$ from the perfect Bayesian equilibrium profile. Suppose that the beliefs in the perfect Bayesian equilibrium are such that all citizens $z \in X$ believe that they are strictly better off from this joint deviation. Then, we say that there is a profitable joint deviation from the perfect Bayesian equilibrium. In the remainder of the paper, we will restrict attention only to those perfect Bayesian equilibria which do not admit any profitable joint deviations. Since no confusion arises, we will refer to this equilibrium concept simply as a "Bayesian equilibrium."

Finally, let us formalize the concept of "implementation." Consider a democratic mechanism $M$. Suppose that for every $P \in \mathcal{P}$, the Bayesian game $(M, P)$ admits a Bayesian equilibrium such that playing the equilibrium strategies leads to the implementation of the Condorcet winner in every state with probability one. Then, we say that the mechanism $M$ implements the Condorcet winner.

\section{Voting with prior communication}

\subsection{Agenda-setter incentives}

One feature of democratic mechanisms is that proposals are endogenous, that is, they are made by a randomly appointed agenda-setter rather than by an outside planner. It is straightforward that a democratic mechanism cannot generally implement the Condorcet winner without giving a tax privilege at least to the one citizen who acts as the agendasetter. In order to see this, suppose that the true state is $k$, but the randomly chosen agenda-setter has a type $z<c\left(q_{k}\right) / q_{k}$. The joint probability of these two events is strictly positive. Such an agenda-setter would rather propose $q_{0}$ and thereby enforce the status quo.

Even if one tried to circumvent this problem by disallowing the proposal $q_{0}$, such an agendasetter could propose $q_{1}$ and then the resulting public good level would be either $q_{1}$ or $q_{0}$, but due to the positive probability of the true state being $k \geq 2$, implementation would fail. Moreover, holding a finite sequence of votes and appointing a new agenda-setter each time does not solve this problem: The probability that every agenda-setter is of some type $z<c\left(q_{k}\right) / q_{k}$ remains strictly positive for any finite number of agenda-setters. Notice that 
this argument would hold even if the agenda-setter knew the true state. This means that to achieve implementation of the Condorcet winner, we must always give an (ex post) unequal treatment to the agenda-setter, no matter how we ensure that the state is revealed. We will focus on the incentives to reveal the state in the sequel of the text.

\subsection{Prior communication mechanism}

In the previous subsection, we have already argued that the implementation of the Condorcet winner through a democratic mechanism requires some distortion of the agendasetter's preferences. In particular, we have considered the possibility of exempting the agenda-setter from the tax, and thus from any contribution to the costs of public good provision. As a consequence, even an agenda-setter of a low type $z<c\left(q_{k}\right) / q_{k}$ who knows that the true state is $k$ would not have an incentive to propose a quantity $q<q_{k}$. However, the tax-exemption may lead to the opposite problem of "over-provision," that is, the tax-exempt agenda-setter is still not interested in proposing the Condorcet winner but in obtaining a high public good level. This reasoning is at the heart of an impossibility result in Lemma 5.2 below.

If we restrict attention to those public good problems which have the distance property as defined in Definition 2.2, then such over-provision does not occur, and hence a tax-exempt agenda-setter would find it optimal to propose the quantity which he believes to be the Condorcet winner. However, in that case, a subgroup of the population is interested in misrepresenting information about its types in such a way that the agenda-setter is misled in his beliefs about the state. This is shown in a second impossibility result in Lemma 5.4 below.

We can conclude that in order to obtain a general implementation result and satisfy the desiderata for democratic mechanisms, we need to make two "interventions" in the preferences of the citizens: One is the tax-exemption for the agenda-setter and the other is some incentive for at least a subgroup of the population to reveal the state of nature prior to the actual decision. This idea is further developed in Sections 6 and 7 below.

In this section, we give two negative results. We consider a mechanism which consists of two stages. The second stage is used to make a decision, while the first stage consists of prior communication. This prior communication is pure cheap talk, and in this sense, it is 
similar to polling. ${ }^{9}$

Prior communication mechanism. In the first ("communication") stage, each citizen sends a positive ("Yes") or negative ("No") signal. ${ }^{10}$ The share of the society which sends the positive signal becomes common knowledge. In the second ("voting") stage, an agendasetter is chosen randomly. The agenda-setter proposes some quantity $q \in Q$. If the majority votes in favor of $q$, then $q$ is implemented, otherwise the status quo $q_{0}$ remains in effect. The agenda-setter is exempted from taxation.

As a result of the tax-exemption, the agenda-setter of type $z$ obtains the utility

$$
u_{e}(z, q)=z q
$$

The prior communication mechanism clearly satisfies the Minimal Message Space, Endogenous Proposals, and Majority Voting requirements. It is also procedurally efficient - the population is active only twice, no matter how large the number of states and alternatives might be. Equal Treatment is curtailed by the tax exemption of the agenda-setter. We argue that this is curtailment is "minor" in four ways: First, given that all citizens have the same probability of becoming an agenda-setter, they are treated equally ex ante. Second, the violation of equal treatment can be seen as minor in the sense that almost all citizens are treated equally, and only a degenerate subset of the population is treated differently. Third, due to the fact that the tax-exempt agenda-setter has zero mass, the exemption has no effect on the tax burden of all other citizens, and therefore "does not do harm." Fourth, one could reduce the agenda-setter's privilege by charging him a fixed lump sum contribution below the expected tax burden.

\footnotetext{
${ }^{9}$ In the mechanism under consideration in this section, the entire population is "polled," but the same results would hold if one only polled a representative sample of the population, as long as this sample is of strictly positive mass.

${ }^{10}$ From a strictly formal point of view, the signals are completely abstract and are intrinsically meaningless. Citizens coordinate tacitly on their meaning. This might seem somewhat artificial. In order to have a more natural interpretation of prior communication, one could imagine that a randomly appointed citizen literally asks the others, "Is your type higher than $\bar{z}$ ?", where $\bar{z} \in Z$ is such that $F_{1}(\bar{z})>F_{2}(\bar{z})>\ldots>F_{n}(\bar{z})$. All citizens respond to this question by saying "Yes" or "No." If all citizens answer truthfully, and the state of nature is $k \in N$, then the share of "Yes"-signals is $1-F_{k}(\bar{z})$.
} 


\subsection{Equilibrium analysis}

For the analysis of the prior communication game, we first consider subgames which consist only of the final votes cast by all citizens. To be more specific, consider a situation where some proposal $q \in Q$ is put to a popular vote against the status quo $q_{0}$. Given that the choice between $q$ and $q_{0}$ is binary and final, there are no gains from voting strategically. Therefore, the voting behavior of each citizen in such a subgame is sincere, which means that they vote in favor of the proposed alternative if and only if they strictly prefer it to the status quo.

Lemma 5.1 All citizens vote sincerely in the voting stage of a prior communication game. That is, citizen $z$ votes in favor of the proposal $q$ if $z>c(q) / q$.

One implication of sincere voting is that the Condorcet winner is accepted in the voting stage if the agenda-setter proposes it. Given that there is no strategic behavior at the voting stage, we can focus attention on a reduced version of the prior communication game. A strategy profile in this reduced game consists only of a communication strategy $\sigma: Z \rightarrow\{Y, N\}$ and a proposal strategy $\rho: Z \times[0,1] \rightarrow Q$, while voting is understood to be sincere by Lemma 5.1. The communication strategy $\sigma$ specifies for each type whether to send a positive or negative signal, and the proposal strategy specifies for each type of the agenda-setter and for each observed share of positive signals the proposal to be made. In the present section, we give two results on the non-existence of Bayesian equilibria which implement the Condorcet winner. These impossibility results are proved by contradiction, and the arguments in the proofs do not make use of the beliefs. Therefore, we need not specify the beliefs associated with the profile $(\rho, \sigma)$ at this stage. Of course, we will have to specify complete profiles of strategies and beliefs when we claim existence of Bayesian equilibria in later sections of the paper.

Lemmata 5.2 and 5.4 give two impossibility results. They show, in particular, that there can be strategic behavior both in the communication of signals and in the choice of the proposal. Lemma 5.2 is relevant in the case where, loosely speaking, there are many feasible public good levels which are not too different from each other, implying that the distance property is violated. In that case, a tax-exempt agenda-setter has an incentive to propose a public good level which is somewhat higher than the Condorcet winner but still low enough to be preferred to the status quo by the majority. 
Lemma 5.2 Suppose there is a public good problem $P \in \mathcal{P}$ such that $\psi_{+}\left(q_{i}\right)<\tilde{q}_{i}$ for some state $i \in N$. Then, the prior communication mechanism does not implement the Condorcet winner.

Proof. Suppose there is a public good problem $P \in \mathcal{P}$ such that in some state $i$, we have $\psi_{+}\left(q_{i}\right)<\tilde{q}_{i}$. The proof follows from a contradiction. Indeed, suppose that there exists a Bayesian equilibrium with strategy profile $\left(\rho^{*}, \sigma^{*}\right)$ of the prior communication game in which the Condorcet winner is implemented in every state. The signaling strategy $\sigma^{*}$ yields a vector $\left(\delta_{k}^{*}\right)_{k \in N}$ of vote shares such that a share $\delta_{k}^{*}$ of the voters sends the positive signal in state $k$. Due to the supposition that the Condorcet winner is implemented, we have $\rho^{*}\left(z, \delta_{k}^{*}\right)=q_{k}$ for every $k \in N$ and $z \in Z$. Since $\psi_{+}\left(q_{i}\right)<\tilde{q}_{i}$, the agenda-setter could make a profitable unilateral deviation from $\left(\rho^{*}, \sigma^{*}\right)$ by proposing $\psi_{+}\left(q_{i}\right)$ instead of $q_{i}$ upon observing $\delta_{i}^{*}$. This proposal will be accepted due to sincere voting in state $i$. Thus, if the true state is $i$, the deviation will improve the agenda-setter's payoff from $z q_{i}$ to $z \psi_{+}\left(q_{i}\right)$. Since $p_{i}>0$, we have obtained the desired contradiction.

The impossibility demonstrated in Lemma 5.2 above is clearly driven by the assumption that some of the relevant public good problems in $\mathcal{P}$ do not satisfy the distance property. One might now wonder whether an implementation result holds if we restrict attention to those public good problems which do have the distance property as in Definition 2.2. In the remainder of this section, we will address this question and show that an impossibility persists. In particular, it turns out that with the distance property, there are incentives for strategic behavior at the communication stage. In particular, those citizens with a sufficiently low type have an incentive to manipulate the information aggregation in such a way that the agenda-setter believes in a state which is higher than the actual state. If the agenda-setter is misled in this way, he proposes an over-provision of public good, but such a proposal is rejected. Hence, the status quo prevails and no public good is produced, which is what the citizens of a sufficiently low type prefer.

In order to make this argument more precise, we introduce the following preliminaries. Let the set of types who prefer $q_{0}$ over $q_{1}$ be denoted by

$$
Z_{-}=\left\{z \in Z \mid z<c\left(q_{1}\right) / q_{1}\right\}
$$

It follows from Assumption 2.1 that the set $Z_{-}$is non-empty and has strictly positive mass. Consider any $q^{\prime}, q^{\prime \prime} \in Q$, such that $q^{\prime \prime} \geq q^{\prime} \geq q_{1}$. Due to the convexity of $c(q)$, preferences over public good levels are single-peaked, and we have that $q^{\prime} \succcurlyeq_{z} q^{\prime \prime}$ for all $z \in Z_{-}$. 
Moreover, it holds by construction that $q_{0} \succcurlyeq_{z} q$ for all $q \in Q \backslash\left\{q_{0}\right\}$ and all $z \in Z_{-}$. Ignoring any public good levels $q \in Q$ such that $q_{0}<q<q_{1}$, this means that types in $Z_{-}$consistently prefer "less to more" of the public good. Now we define a difference function which captures the vertical distance between two consecutive cumulative distribution functions as

$$
d_{k}(z):=F_{k}(z)-F_{k+1}(z), k \in N \backslash\{n\} .
$$

Since cumulative distribution functions are assumed to be twice continuously differentiable, the difference function $d_{k}$ is also twice continuously differentiable. We have assumed the monotonicity of likelihood ratios in the family of probability distributions associated with $\left(F_{k}\right)_{k \in N}$. A simple geometric argument can be used to show that this assumption implies the single-crossing property of the density functions $f_{k}$ and $f_{k+1}$ for every $k \in N \backslash\{n\}$. As a consequence, for every $k \in N \backslash\{n\}$, there is a unique maximizer $z_{k}^{*} \in Z$ of the difference function $d_{k}$. For every $z \in Z$ such that $z<z_{k}^{*}$, the function $d_{k}$ is monotonically increasing, while it is monotonically decreasing for all $z \in Z$ such that $z>z_{k}^{*}$.

Definition 5.3 In a public good problem $P \in \mathcal{P}$, the state of nature is concealable if $\psi_{+}\left(q_{0}\right)=q_{1}$, and, moreover, there is a state $k \in N \backslash\{n\}$ such that $z_{k}^{*} \in Z_{-}$.

From now on, for ease of notation, we write $d_{k}^{*}$ for $d_{k}\left(z_{k}^{*}\right)$ for every $k \in N \backslash\{n\}$. We will show that the Condorcet winner cannot be implemented in those public good problems for which the state is concealable. Because we want to find a mechanism which implements the Condorcet winner for the entire set $\mathcal{P}$ of public good problems, this amounts to an impossibility result.

Lemma 5.4 Suppose that some $P \in \mathcal{P}$ is such that the distance property is satisfied and the state is concealable. Then, the prior communication mechanism does not implement the Condorcet winner.

Proof. The proof is relegated to the appendix.

Lemma 5.4 shows that an impossibility arises even if one restricts attention to public good problems with the distance property. In particular, we have demonstrated the impossibility for public good problems which have the distance property and in which the state is concealable as defined in Definition 5.3. One potential objection which may be raised against Lemma 5.4 is that it might be "vacuous" in the following sense: It might be the case that there do not exist public good problems in which the state is concealable, or at least that the state cannot be concealable in "simple" public good problems with few states 
of nature or few alternatives. In order to demonstrate that Lemma 5.4 is not vacuous in that sense, we now give a very simple example with only two states of nature and only two alternatives in which the state is concealable.

Example 5.5 Let $c(q)=q^{2}+(\alpha+\varepsilon) q$, where $0<\alpha<1 / 2$ and $\varepsilon>0$. Let the type space be $Z=[\varepsilon, 1+\varepsilon]$, and let the cumulative distribution functions be given by $F_{1}(z)=z-\varepsilon$, and $F_{2}(z)=(z-\varepsilon)^{\nu}$, where we need to assume $\nu>1$ to ensure first-order stochastic dominance. Using the equality $F_{k}\left(c^{\prime}\left(q_{k}\right)\right)=1 / 2$, we can find the Condorcet winners $q_{1}=$ $\frac{1-2 \alpha}{4}$ and $q_{2}=\frac{(1 / 2)^{(1 / \nu)}-\alpha}{2}$. We assume that $Q=\left\{q_{0}, q_{1}, q_{2}\right\}$. We can verify that the function $F_{1}(z)-F_{2}(z)$ attains a unique maximum at the point $z=(1 / \nu)^{\left(\frac{1}{\nu-1}\right)}+\varepsilon$. The state is concealable if $(1 / \nu)^{\left(\frac{1}{\nu-1}\right)}<\frac{1}{2} \alpha+\frac{1}{4}$. Now we see that there is a non-degenerate set of values for $\alpha$ and $\nu$ such that the state is concealable. One example is $\alpha=0.4$ and $\nu=1.2$.

In principle, one may of course wonder if the prior communication mechanism can accomplish the implementation of the Condorcet winner if we restrict the domain of public good problems even further, that is, if we require the distance property and exclude concealable states.

Even on such a heavily restricted domain of public good problems, however, implementation may still fail. In order to get an intuitive idea why implementation can still fail, consider a public good problem which belongs to this restricted domain and suppose that there is a type $\tilde{z} \in Z$ and a state $k \in N$ such that $z_{k}^{*}<\tilde{z}<c\left(q_{k}\right) / q_{k}$. Suppose that all types $z \in Z$ with $z<\tilde{z}$ coordinate in order to misrepresent state $k$ as state $k+1$. This is beneficial for them if state $k$ is the true state. In the absence of the concealable property, it is possible, however, that this misrepresentation is harmful to citizen $z<\tilde{z}$ if the true state is some state other than $k$. Hence, we cannot say unambiguously whether such misrepresentation is beneficial or harmful in general, but it is certainly beneficial when the prior probability $p_{k}$ is sufficiently high.

\subsection{Summary and discussion}

In conclusion, we see that a prior communication mechanism cannot generally implement the Condorcet winner on the entire domain $\mathcal{P}$ of public good problems. Moreover, this impossibility persists even if we heavily restrict the public good problem by imposing the distance property, or by requiring that states be not concealable. We summarize this result in the following theorem. 
Theorem 5.6 A prior communication mechanism does not generally implement the Condorcet winner, irrespective of whether the distance property holds.

In the prior communication mechanism, the agenda-setter is chosen randomly among all citizens. One may find it undesirable that citizens can be appointed agenda-setter without their consent. To alleviate this objection, we could modify the mechanism by saying that each citizen can decide whether to "apply" for the role of agenda-setter, and the actual agenda-setter is then randomly chosen among all "applicants." Our analysis is robust to such a modification. First, one can show that an individual citizen is not worse off from being the agenda-setter. Second, one can show that there can never be an equilibrium in which there is no agenda-setter. In order to see this, observe that without an agendasetter, the outcome would always be $q_{0}$. Next consider a citizen $z$ who deviates from a strategy profile in which no citizen wants to be an agenda-setter by applying for agendasetting, and then always proposing $q_{1}$. Due to sincere voting, $q_{1}$ is accepted irrespective of the actual state. The resulting payoff for the agenda-setter would be $z q_{1}$, and since $z>0$, his deviation would be profitable compared to the situation where there is no agenda-setter and all citizens therefore obtain a zero payoff.

The distinction between public good problems with the distance property and the entire domain $\mathcal{P}$ of public good problems is important again in the next two sections. In Section 6 , we introduce a mechanism which implements the Condorcet winner on the restricted domain of public good problems with the distance property. In Section 7, we modify that mechanism in such a way that it achieves implementation on the whole domain $\mathcal{P}$ of public good problems.

\section{Information revelation by a privileged sample}

\subsection{Definition and discussion of the mechanism}

In this section, we present a mechanism which is similar to the prior communication mechanism in Section 5, except that the prior communication is done only by a small random sample of the society whose members exempted from taxation. The prior communication by a small random sample is reminiscent of polling. However, the distortion of sample members' preferences through a tax-exemption makes this approach different from polling. We call this mechanism prior communication with sampling (PCS). For the PCS mech- 
anism, we find a first existence result: The Condorcet winner is implemented for those public good problems which satisfy the distance property. The PCS mechanism requires only one popular vote (and one round of prior communication by the small sample group) In Section 7, we will introduce a modification of the PCS mechanism which requires two popular votes (and one round of prior communication by a sample group) to implement the Condorcet winner irrespective of the distance property.

The main idea behind the PCS mechanism is that the tax-exemption motivates all members of the small sample group to reveal information about their types truthfully, whatever those types may be. As in the prior communication mechanism in Section 5, the prior communication affects the outcome of the mechanism only through its effect on the beliefs - it is "pure cheap talk." A quantity $q \in Q \backslash\left\{q_{0}\right\}$ can only become the outcome of the mechanism if it directly defeats the status quo $q_{0}$ in a popular vote with a super-majority rule. This super-majority rule is such that it requires a simple majority of all citizens outside the sample group.

Mechanism with prior communication by a sample group. A subset of mass $\lambda(0<\lambda<1)$ is randomly drawn from the population, we call it the sample group. At the first ("communication") stage, each member of the sample group takes a binary decision to send either a positive or a negative signal. ${ }^{11}$ The share of the sample group members who have sent the positive signal becomes common knowledge. In the second ("voting") stage, an agenda-setter is randomly drawn from the whole society and proposes some $q \in Q$. The society votes on $q$ versus $q_{0}$ by a super-majority rule: The proposal $q$ is implemented if at least a share $(1+\lambda) / 2$ of the population accept it. ${ }^{12}$ Otherwise, the status quo $q_{0}$ remains in effect. Sample group members, as well as the agenda-setter are tax-exempt, while all other citizens are taxed uniformly.

The PCS mechanism clearly satisfies the Minimal Message Space, Endogenous Proposals, and Majority Voting requirements. ${ }^{13}$ This mechanism also does very well in terms of Procedural Efficiency. The whole population is active only once. In addition, there is prior

\footnotetext{
${ }^{11}$ Again, as in the prior communication mechanism, the citizens can reveal information by coordinating on saying "Yes" if and only if their type is higher than some critical type, and saying "No" otherwise.

${ }^{12} \mathrm{We}$ want to require that a simple majority of the citizens outside the sample group need to accept the proposal, even if all sample group members accept. Thus, the super-majority is $\lambda+(1-\lambda) / 2=(1+\lambda) / 2$.

${ }^{13}$ According to the desideratum of Majority Voting, the status quo can only be replaced with some feasible alternative if a simple majority votes in favor of the alternative. This requirement is satisfied when a super-majority rule is used.
} 
communication by the small sample group. This is irrespective of the number of states and the number of public good levels. The drawback of the PCS mechanism is the unequal tax treatment of the sample group. Contrary to a tax-exemption for agenda-setters only, the tax-exemption for a sample of strictly positive mass has an effect on the tax burden of the remaining population.

Nevertheless, some arguments why the tax-exemption should only be seen as a minor curtailment of the Equal Treatment desideratum remain valid also with respect to the PCS mechanism: First, it is still true that all citizens are treated equally ex ante because every citizen has equal probability of becoming a sample group member. Second, while the mass of the sample group is strictly positive, it can be made arbitrarily small. Third, this also implies that the effect of the sample's tax privilege on the tax burden of the remaining population can be arbitrarily small. Moreover, the super-majority requirement inherent to the PCS mechanism ensures that the sample group cannot advance its interests against a majority of the citizens outside of the sample group. Finally, exactly the same preferences induced among sample group members over the different public good levels could alternatively be induced by treating all citizens equally with regard to taxation but granting sample group members a subsidy conditional on the final outcome of the mechanism. Gersbach et al. (2013) point out that constitutional equal treatment clauses do not apply to subsidization.

In the next subsection, we will give an implementation result. The PCS mechanism implements the Condorcet winner for all those public good problems which have the distance property. In Section 7, we will discuss a modified version of the PCS mechanism which extends the implementation result to the entire domain $\mathcal{P}$ of public good problems irrespective of the distance property.

\subsection{Existence result}

In this subsection, we show that the PCS mechanism implements the Condorcet winner in those public good problems which satisfy the distance property. For this analysis, we need the following preliminaries:

Contrary to the analysis in the previous section, under the PCS mechanism, the public good is no longer financed by uniform taxation. From the point of view of citizens outside the sample group, the provision of a public good quantity $q \in Q$ is no longer associated 
with a tax burden of $c(q)$, but of $\left(\frac{1}{1-\lambda}\right) c(q)$. Consequently, the utility of citizen $z$ outside the sample group from the public good level $q \in Q$ is given by

$$
\hat{u}(z, q)=z q-\left(\frac{1}{1-\lambda}\right) c(q) .
$$

Just as in the prior communication mechanism of Section 5, the popular vote in the PCS mechanism is a binary and final choice and thus strategic voting can never be beneficial. Analogously to Lemma 5.1, the citizens vote sincerely in the PCS mechanism. We now formally spell out the sincere voting rule in the PCS mechanism. Of course, this rule is different for sample group members than for regular citizens. Indeed, suppose that at the voting stage of the PCS mechanism the alternative $q \in Q$ is pitted against the status quo $q_{0}$. Since $Z \subset \mathbb{R}_{++}$, we have that $z q>0$ for every $q \in Q \backslash\left\{q_{0}\right\}$, and thus all sample group members vote sincerely in favor of the proposal. Citizen $z$, who is not a sample group member, prefers $q$ to $q_{0}$ if

$$
z>\frac{c(q)}{(1-\lambda) q}
$$

For every state $i \in N$, define $\tilde{q}_{i}^{\lambda}$ as the solution to the equality

$$
F_{i}\left(\frac{c\left(\tilde{q}_{i}^{\lambda}\right)}{(1-\lambda) \tilde{q}_{i}^{\lambda}}\right)=1 / 2 .
$$

In words, if the true state is $i$, then a majority of citizens outside the sample group prefers any quantity $q \in Q$ with $q<\tilde{q}_{i}^{\lambda}$ over $q_{0}$, and prefers $q_{0}$ to any quantity $q \in Q$ such that $q>\tilde{q}_{i}^{\lambda}$. The assumptions introduced on the cumulative distribution functions $\left(F_{i}\right)_{i \in N}$ guarantee that $\tilde{q}_{i}^{\lambda}$ exists.

For every $\lambda>0$, we have $\tilde{q}_{i}^{\lambda}<\tilde{q}_{i}$, and we find that $\tilde{q}_{i}^{\lambda}$ converges to $\tilde{q}_{i}$ as $\lambda \downarrow 0$.

Lemma 6.1 Suppose that $q \in Q$ is a proposal which is preferred by the majority to $q_{0}$ under uniform taxation. Then, $q$ is also preferred to $q_{0}$ by the majority under the tax-exemption, provided that $\lambda>0$ is small enough.

The tax-exemption for a small sample group is meant to distort the preferences of sample group members in such a way that they prefer more to less of the public good. While this tax-exemption may in principle also distort the preference rankings of citizens outside the sample group, we have now established that such distortions do not occur when the size of the sample group is sufficiently small. This finding is due to the assumption that the set of feasible alternatives is discrete. 
The PCS mechanism combined with a public good problem $P \in \mathcal{P}$ constitutes the prior communication with sampling (PCS) game. By the same logic as in the previous section, citizens vote sincerely at the voting stage, so that it suffices to consider a reduced PCS game without the voting stage. Then, the notions of strategies, beliefs, and the equilibrium concept introduced in Section 4 can be specialized to PCS game as follows:

A communication strategy for the sample group is a map $\sigma: Z \rightarrow\{Y e s, N o\}$ which indicates for each type of a sample group member whether he sends a positive or negative signal. A belief $\pi: Z \times[0,1] \rightarrow \Delta_{n}$ indicates the subjective probabilities attached to the individual states for each type $z \in Z$ and for the observed share of positive signals. A proposal strategy for the agenda-setter is a map $\rho: Z \times[0,1] \rightarrow Q$ indicating the proposal to be made, given the type of the agenda-setter and the observed share of sample group members who have sent the positive signal. For a subset of sample group members $X \subset Z$ of strictly positive mass, a joint deviation from a profile $(\pi, \rho, \sigma)$ is some $\hat{\sigma}$ such that $\hat{\sigma}(z)=\sigma(z)$ for all $z \in Z \backslash X$ and $\hat{\sigma}(z) \neq \sigma(z)$ for some $z \in X$. The joint deviation is called profitable if each citizen $z \in X$ obtains a strictly greater payoff under $(\pi, \rho, \hat{\sigma})$ than under $(\pi, \rho, \sigma)$. A profile $\left(\pi^{*}, \rho^{*}, \sigma^{*}\right)$ is a Bayesian equilibrium of the PCS game if there is no profitable joint deviation from $\sigma^{*}$ given $\pi^{*}$ and $\rho^{*}$ and for all types $z \in Z$, the strategy $\rho^{*}$ is optimal, given beliefs $\pi^{*}$ and strategy $\sigma^{*}$, and, finally, the beliefs $\pi^{*}$ are consistent with communication strategy $\sigma^{*}$.

The (appropriately reduced) PCS game is essentially a simple Bayesian game between the sample group members and the agenda-setter. The strategic interaction between them entails no conflict as they all share the same preferences over the possible outcomes. To advance their common interest, they need to accomplish coordination on the meaning of the signals, and thereby allow the dissemination of information. In order to show that the sample group members and the agenda-setter can indeed coordinate their actions successfully, we construct a Bayesian equilibrium which implements the Condorcet winner.

Take any $\bar{z} \in \operatorname{int}(Z)$, and define the strategy $\sigma^{\bar{z}}$ as follows:

$$
\sigma^{\bar{z}}(z)= \begin{cases}Y e s & \text { if } z \geq \bar{z} \\ N o & \text { otherwise }\end{cases}
$$

We associate to the strategy $\sigma^{\bar{z}}$ a belief $\pi^{\bar{z}}$ which is defined as follows: 


$$
\pi^{\bar{z}}(\delta, z)= \begin{cases}e_{1} & \text { if } \delta<1-F_{2}(\bar{z}) \\ e_{k} & \text { if } \delta \in\left[1-F_{k}(\bar{z}), 1-F_{k+1}(\bar{z})\right), k=2, \ldots, n-1 \\ e_{n} & \text { if } \delta \geq 1-F_{n}(\bar{z}) .\end{cases}
$$

Now we introduce the following proposal strategy:

$$
\rho^{\bar{z}}(\delta, z)= \begin{cases}q_{1} & \text { if } \delta<1-F_{2}(\bar{z}), \\ q_{k} & \text { if } \delta \in\left[1-F_{k}(\bar{z}), 1-F_{k+1}(\bar{z})\right), k=2, \ldots, n-1, \\ q_{n} & \text { if } \delta \geq 1-F_{n}(\bar{z}) .\end{cases}
$$

With this construction, we can state a first existence result for truthful information aggregation and implementation of the Condorcet winner.

Theorem 6.2 Consider a PCS game with a public good problem $P$ which has the distance property. Then, for all $\bar{z} \in \operatorname{int}(Z)$, the profile $\left(\pi^{\bar{z}}, \rho^{\bar{z}}, \sigma^{\bar{z}}\right)$ is a Bayesian equilibrium of this PCS game. Thus, if $\mathcal{P}$ is restricted to the public good problems which have the distance property, then the PCS mechanism implements the Condorcet winner.

Proof. Suppose that sample group members play according to $\rho^{\bar{z}}$ and $\sigma^{\bar{z}}$, and beliefs are given by $\pi^{\bar{z}}$. By construction, $\pi^{\bar{z}}$ is consistent with $\sigma^{\bar{z}}$. We show first that the proposal strategy is optimal. Indeed, let $i$ be the true state, and $q_{i}$ be the Condorcet winner. Due to the distance property and sincere voting, any proposal $q>q_{i}$ will be rejected, and any proposal $q<q_{i}$ will be accepted in the popular vote. Since the agenda-setter is tax-exempt, it follows immediately that it is optimal for him to propose the quantity $q_{i}$ whenever his belief is $e_{i}$. Otherwise, the agenda-setter makes any proposal $q \in Q \backslash\left\{q_{i}\right\}$, the outcome of the mechanism is some $q^{\prime} \in\left\{q, q_{0}\right\}$, and $q^{\prime}<q_{i}$. Next we show that the communication strategy is optimal. All members of the sample group are tax-exempt. A joint deviation for a subset of the sample group could only be profitable if it led to some public good level $q>q_{i}$. Due to sincere voting, such a public good level will not be implemented. 


\subsection{Discussion}

A game based on the PCS mechanism admits a multitude of equilibria which implement the Condorcet winner. In fact, if the cumulative distribution functions are such that $F_{1}(z), \ldots, F_{n}(z)$ are $n$ distinct numerical values for every $z \in \operatorname{int}(Z)$, then every interior type can serve as the threshold type $\bar{z}$ used in Theorem 6.2 and is thus associated with one revealing Bayesian equilibrium. This multiplicity of equilibria can be viewed as a somewhat problematic feature of the mechanism. After all, the implementation of the Condorcet winner hinges on the ability of the players to coordinate on one particular threshold type $\bar{z}$. The mechanism includes no "communication device" to accomplish this coordination. As hinted at before, however, the PCS mechanism can easily be modified and extended to facilitate the coordination of sample group members. Since all sample group members are interested in the revelation of the state, there is no inherent obstacle to coordination. For instance, one could allow one member of the sample group to send a message from within a rich message space before the positive or negative signals are sent. This could be interpreted as one sample group member asking, "Is your type higher or lower than $\bar{z}$ ?"

We note that the existence theorem does not depend on the prior belief of citizens about the probability distribution $p$ on the states. Hence, the mechanism with experimentation is prior-free, which is an important and desirable robustness property of the mechanism. ${ }^{14}$

The Bayesian equilibrium of Theorem 6.2 above is similar to a separating equilibrium in a signaling game. Next to a separating equilibrium, signaling games typically admit a pooling equilibrium in which the signal is uninformative. This logic carries over to the situation at hand. There clearly exists a Bayesian equilibrium in which all sample group members send the positive signal irrespective of their types, and the agenda-setter of type $z$ has belief $\beta(z)$ and proposes that quantity which is optimal given the belief $\beta(z)$, completely disregarding the messages of the sample group members. However, such a pooling equilibrium can be eliminated by suitable refinements of the equilibrium concept. In particular, it can be shown that all sample group members and the agenda-setter expect a strictly greater payoff from playing the separating equilibrium than from playing a pooling equilibrium, irrespective of their types.

The main idea behind the PCS mechanism is simple: On the one hand, the tax-exemption

\footnotetext{
${ }^{14}$ For the general theory of robust mechanisms in the standard framework, we refer to Bergemann and Morris (2005).
} 
gives sample group members and the agenda-setter an incentive to aim at the highest possible public good level. On the other hand, the distance property discourages the sample group members from any attempt to target a public good level higher than the Condorcet winner. Therefore, it seems intuitive that the existence result in Theorem 6.2 is limited to the public good problems which satisfy the distance property. However, we will show in the next section that a modified version of the PCS mechanism accomplishes implementation without relying on the distance property at all. This modified mechanism requires one extra popular vote. Hence, we have an implementation result on the entire domain $\mathcal{P}$ of public good problems.

\section{$7 \quad$ A general implementation result}

\subsection{Prior sampling and selection}

In this section, we give the main existence result. It says that the Condorcet winner can be implemented for all public good problems in $\mathcal{P}$ by a mechanism which we call prior communication with sampling and selection (PCSS) mechanism. This mechanism is very similar to the PCS mechanism in the previous section, with three additions. First, an additional popular vote ("selection stage") is required. Second, the privileged group might have to receive a cash transfer on top of the tax-exemption. Third, these privileges are granted only "conditionally," in a sense to be made precise in the definition of the mechanism.

Prior communication with sampling and selection. A sample group of size $\lambda(0<$ $\lambda<1$ ) is drawn randomly from the population. In the first ("communication") stage, each sample group member sends a binary signal. The share of sample group members who have sent the affirmative signal becomes common knowledge. Now an agenda-setter is randomly drawn from the society. This agenda-setter proposes some $q \in Q$. If $q=q_{0}=0$, then the procedure ends and the status quo $q_{0}$ remains in effect. If the agenda-setter proposes $q=\psi_{+}\left(q_{0}\right)$, then the procedure moves immediately to popular vote between $q_{0}$ and $\psi_{+}\left(q_{0}\right)$, the winner is the outcome of the mechanism. If the agenda-setter proposes some

$q \in Q \backslash\left\{q_{0}, \psi_{+}\left(q_{0}\right)\right\}$, then the procedure moves to a second ("selection") stage, where a popular vote is held between $q$ and $\psi_{-}(q)$. The proposal $q$ wins the selection stage if and only if it obtains at least the share $(1+\lambda) / 2$ of the votes. Otherwise, the amendment $\psi_{-}(q)$ 
is considered the winner of the selection stage. Finally, in the third ("voting") stage, the winner of the popular vote in the selection stage is brought to a popular vote against $q_{0}$. In the voting stage, the winner of the selection stage needs at least $(1+\lambda) / 2$ of the votes to be implemented if it is the proposal $q$ or at least the share $1 / 2$ if it is the amendment $\psi_{-}(q)$. Otherwise, the status quo $q_{0}$ remains in effect. If and only if the proposal $q$ made by the agenda-setter wins a popular vote against $q_{0}$, then the agenda-setter, as well as the sample group members, are tax-exempt and receive a transfer of $\mu$. Neither a tax-exemption nor a transfer are given if the final outcome of the mechanism is either the status quo $q_{0}$ or the amendment $\psi_{-}(q)$.

Repeating the same arguments as in Sections 5 and 6, we can point out that the voting stage of the PCSS mechanism is a final and binary choice which leaves no room for profitable strategic behavior. As a result, citizens vote sincerely at the voting stage. Contrary to the PCS mechanism discussed in Section 6, sample group members and the agenda-setter not only receive a tax-exemption but also a per-capita transfer $\mu$. Both of these privileges are conditional on final acceptance of the proposal. The sincere voting rule changes accordingly. Indeed, suppose that at the voting stage, the alternative $q \in Q$ is pitted against the status quo $q_{0}$. Consider first the case where $q$ is not the original proposal, but the amendment. In that case, neither tax-exemptions nor transfers will be granted, so that sincere voting simply means that citizen $z$ votes for $q$ if $z>c(q) / q$. In the other case, $q$ is the original proposal made by the agenda-setter. Since $z q>0$ for all $q \in Q \backslash\left\{q_{0}\right\}$ and all $z \in Z$, it is sincere for every sample group member to vote in favor of $q$. Citizen $z$, who is not a sample group member, votes sincerely in favor of $q$ if

$$
z>\frac{c(q)+\lambda \mu}{(1-\lambda) q}
$$

For every state $i \in N$, define $\tilde{q}_{i}^{(\lambda, \mu)}$ as the solution to the equality

$$
F_{i}\left(\frac{c\left(\tilde{q}_{i}^{(\lambda, \mu)}\right)+\lambda \mu}{(1-\lambda) \tilde{q}_{i}^{(\lambda, \mu)}}\right)=1 / 2 .
$$

We note that $\tilde{q}_{i}^{(\lambda, \mu)}<\tilde{q}_{i}$ for $\lambda>0$ and $\mu \geq 0$, and, moreover, for any value of $\mu \geq 0$, we find that $\tilde{q}_{i}^{(\lambda, \mu)}$ converges to $\tilde{q}_{i}$ as $\lambda \downarrow 0$.

Remark. In Section 6, we have justified the tax exemption for the sample group by saying, among other arguments, that the cost which citizens outside the sample group incur in 
order to finance the sample group members' tax exemption vanishes when the size $\lambda$ of the sample group becomes sufficiently small. We note that this argument extends to the transfer $\mu$. The per-capita transfer $\mu$ paid to sample group members comes at a per-capita cost of $(\lambda \mu) /(1-\lambda)$ to citizens outside the sample group. Clearly, this cost vanishes in the limit as $\lambda \downarrow 0$, for any finite value of $\mu>0$.

\subsection{The implementation result}

By the same arguments as in the prior communication and PCS mechanisms, it is also true under the PCSS mechanism that the final popular vote in the "voting stage" leaves no room for profitable strategic voting. As a consequence, voting is sincere in the final stage of the PCSS mechanism, and therefore, we can consider the pair of the PCSS mechanism and a public good problem $P \in \mathcal{P}$ as a reduced PCSS game, in which the following strategies and beliefs are relevant: A communication strategy $\sigma: Z \rightarrow\{Y, N\}$ specifies a positive or negative signal for each type. A map $\pi: Z \times[0,1] \rightarrow \Delta^{n}$ assigns a belief about the state to each type and each observed share of positive signals. A proposal strategy $\rho: Z \times \Delta^{n} \rightarrow Q$ specifies a proposal for each type of the agenda-setter and each belief about the states. This definition of the communication and proposal strategies as well as the beliefs is perfectly analogous to those introduced in the context of the PCS mechanism in Section 6.

The PCSS game in general involves two successive popular votes. There are two exceptions from this general rule: There is only one popular vote if the agenda-setter's proposal is $\psi_{+}\left(q_{0}\right)$, and there is no popular vote if the agenda-setter proposes $q_{0}$. In all other cases, there is a selection stage prior to the voting stage, between a proposal $q \in Q \backslash\left\{q_{0}, \psi_{+}\left(q_{0}\right)\right\}$ and the amendment $\psi_{-}(q)$. A selection strategy is a map $\omega: Z \times \Delta^{n} \times Q \rightarrow\{P, A\}$ which indicates whether a citizen of type $z \in Z$ with belief $\pi \in \Delta^{n}$ selects the proposal $q \in Q \backslash\left\{q_{0}, \psi_{+}\left(q_{0}\right)\right\}$ or the amendment $\psi_{-}(q)$ at the selection stage. We denote by $\omega_{s}$ a selection strategy for sample group members and by $\omega_{p}$ a selection strategy for citizens outside the sample group. Assuming that selection strategies $\omega_{s}$ and $\omega_{p}$ are optimal, we are going to examine their restrictions to the case of deterministic beliefs. That is, we are going to describe the optimal selection behavior for a citizen of type $z \in Z$ who believes with probability one that the true state is $i \in N$, and needs to select either a proposal $q \in Q \backslash\left\{q_{0}, \psi_{+}\left(q_{0}\right)\right\}$ or the amendment $\psi_{-}(q)$. In order to construct optimal selection strategies for sample group members and citizens outside the sample group given deterministic beliefs, we need to distinguish the following three cases: 
Case 1: $\psi_{-}(q)>\tilde{q}_{i}^{(\lambda, \mu)}$. In this case, both the amendment and the proposal would be rejected at the voting stage. The selection is inconsequential, so in particular, it is optimal to select the proposal, no matter if one is a sample group member or not.

Case 2: $q \leq \tilde{q}_{i}^{(\lambda, \mu)}$. In this case, both the proposal and the amendment would be accepted at the voting stage. Hence, the selection becomes a binary and "final" choice between the proposal and the amendment. Hence, optimal selection must be sincere selection. A citizen $z$ outside the sample group prefers proposal $q$ over amendment $\psi_{-}(q)$ if $z q-\frac{c(q)+\lambda \mu}{(1-\lambda)}>z \psi_{-}(q)-c\left(\psi_{-}(q)\right)$. A sample group member prefers $q$ over $\psi_{-}(q)$ because $\mu+z q>z \psi_{-}(q)-c\left(\psi_{-}(q)\right)$, that is, a sample group member sincerely selects the proposal ("more to less").

Case 3: $\quad q>\tilde{q}_{i}^{(\lambda, \mu)} \geq \psi_{-}(q)$. In this case, the proposal would be rejected, but the amendment would be accepted at the voting stage. For any citizen $z$, it is optimal to select the proposal $q$ if and only if $z<c\left(\psi_{-}(q)\right) / \psi_{-}(q)$. This is true irrespective of whether citizen $z$ does or does not belong to the sample group.

We have now described optimal selection given deterministic beliefs. For the rest of this section, we denote by $\left(\omega_{s}^{*}, \omega_{p}^{*}\right)$ some pair of optimal selection strategies for sample group members and citizens outside the sample group. In the sequel of our argument, Case 2 above is crucial. In particular, the above construction of $\left(\omega_{s}^{*}, \omega_{p}^{*}\right)$ implies the following statement: If all citizens have the same belief $e_{i}$ and the proposal at the selection stage is some $q \in Q$ with $q \leq \tilde{q}_{i}^{(\lambda, \mu)}$, then all sample group members select sincerely under any optimal selection strategy $\omega_{s}^{*}$, while all citizens outside the sample group select the proposal under any optimal selection strategy $\omega_{p}^{*}$.

We now proceed with the construction of a signaling strategy, a proposal strategy, and some consistent beliefs. Given some $\bar{z} \in \operatorname{int}(Z)$, we take the signaling strategy already familiar from Section 6:

$$
\sigma^{\bar{z}}(z)= \begin{cases}Y e s & \text { if } z \geq \bar{z} \\ N_{o} & \text { otherwise }\end{cases}
$$

Verbally, citizens with a type higher than some threshold $\bar{z}$ send the positive signal, while other citizens send the negative signal. Likewise, the beliefs of the agenda-setter and the 
sample group members correspond to the construction in Section 6 ,

$$
\pi^{\bar{z}}(\delta, z)= \begin{cases}e_{1} & \text { if } \delta<1-F_{2}(\bar{z}) \\ e_{k} & \text { if } \delta \in\left[1-F_{k}(\bar{z}), 1-F_{k+1}(\bar{z})\right), k=2, \ldots, n-1 \\ e_{n} & \text { if } \delta \geq 1-F_{n}(\bar{z})\end{cases}
$$

That is, the agenda-setter and the sample group members "understand" the information revealed by the sample group members, and end up with deterministic beliefs about the state. The agenda-setter's strategy is to propose the quantity which he believes to be the Condorcet winner, hence

$$
\rho^{\bar{z}}(\delta, z)= \begin{cases}q_{1} & \text { if } \delta<1-F_{2}(\bar{z}), \\ q_{k} & \text { if } \delta \in\left[1-F_{k}(\bar{z}), 1-F_{k+1}(\bar{z})\right), k=2, \ldots, n-1, \\ q_{n} & \text { if } \delta \geq 1-F_{n}(\bar{z}) .\end{cases}
$$

Finally, we construct the following belief for the citizens outside of the sample group, which depend on the proposal $q \in Q$ made by the agenda-setter:

$$
\pi^{*}(\delta, q, z)= \begin{cases}e_{1} & \text { if } q<q_{2}, \\ e_{k} & \text { if } q_{k} \leq q<q_{k+1}, k=2, \ldots, n-1, \\ e_{n} & \text { if } q=q_{n} .\end{cases}
$$

We note that citizens outside of the sample group base their beliefs only on the proposal made by the agenda-setter and not directly on the information revealed by the sample group. ${ }^{15}$ Theorem 7.1 is the main existence result of this paper. It claims that the profile

\footnotetext{
${ }^{15}$ It may seem intuitive to use a construction in which all citizens share the belief $\pi^{\bar{z}}$. However, such a construction might not accomplish implementation. In order to see this, consider Case 3 in our construction of the optimal selection strategy. It says that citizens of a sufficiently low type select the proposal at the selection stage if they believe that the proposal will be rejected while the amendment will be accepted. Let $X \subset Z$ have sufficiently low types. Since the agenda-setter belongs to $X$ with strictly positive probability, the group $X$ might want to misrepresent information in such a way that citizens believe the state to be lower than it actually is. If the agenda-setter belongs to $X$, he can then propose a quantity which the citizens believe would be rejected at the voting stage, and which is so high that the majority prefers the status quo to it. The citizens would then select a quantity which they assume will be rejected at the voting state. In fact, however, it will be accepted due to sincere voting, which is beneficial for the high types who make up group $X$. This problem is avoided when we construct beliefs for the citizens outside the sample group which are based only on the proposal.
} 
of strategies and beliefs which we have just constructed is an equilibrium of the PCSS game, and therefore the PCSS mechanism implements the Condorcet winner.

Theorem 7.1 A profile $\left(\pi^{\bar{z}}, \pi^{*}, \rho^{\bar{z}}, \sigma^{\bar{z}}, \omega_{s}^{*}, \omega_{p}^{*}\right)$ of strategies and beliefs is a Bayesian equilibrium of the PCSS game if $\mu$ is sufficiently large. Thus, the PCSS mechanism implements the Condorcet winner if $\mu$ is large enough.

Proof. The proof is relegated to the appendix.

\subsection{Discussion}

Theorem 7.1 above states an implementation result provided that the transfer $\mu$ is sufficiently large. In the proof of Theorem 7.1, it turns out that a strictly positive transfer $\mu>0$ is necessary for implementation only if there are a state $i \in N$ and a quantity $\hat{q}_{i} \in Q$ which satisfy the inequalities

$$
\begin{aligned}
\tilde{q}_{i}>\hat{q}_{i} & >q_{i}, \\
z \hat{q}_{i}-c\left(\hat{q}_{i}\right) & \geq z q_{i},
\end{aligned}
$$

If such $i \in N$ and $\hat{q}_{i} \in Q$ do not exist, then the implementation result of Theorem 7.1 holds even for $\mu=0$. Thus, we have the following corollary:

Corollary 7.2 Suppose that the type space $Z$, the cost function $c$, and the cumulative distribution functions $\left(F_{i}\right)_{i \in N}$ are such that for all $i \in N$ and for all $z \in Z$, it holds that $z \leq c\left(\tilde{q}_{i}\right) /\left(\tilde{q}_{i}-q_{i}\right)$. Then, the PCSS mechanism implements the Condorcet winner with $\mu=0$.

Verbally, the mode of operation of the PCSS mechanism can be described as follows: The tax-exemption ensures that the agenda-setter and all sample group members have the same preference ranking over $Q$. Irrespective of their individual types, they all prefer more to less of the public good if they are tax-exempt. As a consequence, they all have a common interest to aim for the highest public good level which the majority of the entire society prefers to the status quo. If the distance property holds, the highest public good level preferred by the majority to the status quo is the Condorcet winner, and thus implementation is accomplished. If the distance property does not hold, the tax-exemption motivates the agenda-setter and the sample group members to aim for a public good level in excess of the Condorcet winner. In order to counteract the incentive for over-provision 
of the public good, the PCSS mechanism includes the selection stage at which the entire society can make a minimal downward correction of the proposal. Since the tax exemption for the agenda-setter and the sample group is conditional on no such downward correction taking place at the selection stage, the incentive to over-provide public good is eliminated.

Let us evaluate the PCSS mechanism against the desiderata for democratic mechanisms. Clearly, the PCSS mechanism satisfies the Minimal Message Space and Majority Voting desiderata. Moreover, it is very attractive in terms of Procedural Efficiency - it can implement the Condorcet winner in two popular votes irrespective of the number of states or alternatives involved in the public good problem. With regard to the Equal Treatment desideratum, the PCSS mechanism is similar to the PCS mechanism of Section 6. A sample of positive mass receives a tax-exemption and potentially an additional transfer. However, the mass of this sample is arbitrarily small and all citizens have an ex ante equal probability of becoming a sample group member. Contrary to the discussion in Section 6, the PCSS mechanism grants the tax-exemption or the possible transfer only conditionally. That is, we can interpret the privileged treatment as a "reward" for truthful information aggregation since this revelation is socially desirable. Finally, consider the desideratum of Endogenous Proposals. In the description of the PCSS mechanism, we have imposed that any proposal $q \in Q$ is pitted against its "predecessor" $\psi_{-}(q)$ at the selection stage. This might seem at odds with Endogenous Proposals. However, this problem can be avoided by randomly appointing a second ("rival") agenda-setter who chooses the amendment and receives a reward (tax-exemption and transfer) if and only if the amendment is selected and becomes the final outcome of the mechanism. In such a model, one can show that there is a Bayesian equilibrium in which the rival agenda-setter always believes with probability one that his proposal is the Condorcet winner and always suggests the predecessor of the proposal as an amendment. The existence of such an equilibrium follows from the same logic as the proof of Theorem 7.1 .

One important extension of our model is to ask whether the implementation of the Condorcet winner can be accomplished if the status quo is not $q_{0}$ (zero public good provision), but some other public good level $\bar{q} \in Q \backslash\left\{q_{0}\right\}$. If this is the case, then the implementation result of Theorem 7.1 satisfies a kind of "dynamic robustness" property: If the state of nature changes over time, then the PCSS mechanism can be applied again in order to move from the "old" Condorcet winner to the "new" Condorcet winner. We conjecture that, indeed, the PCSS mechanism can be suitably modified implement the Condorcet winner 
for any given status quo.

To see why this would be the case, let us define an $(\eta, \theta)$-tax treatment as specifying that a citizen receives a lump sum transfer of $\theta$ and needs to pay a $\operatorname{tax} \eta c(q)$ when the public good level is $q \in Q$, and If $\eta>1$ is chosen sufficiently high, then a citizen of any type $z \in Z$ who is subjected to an $(\eta, \theta)$-tax treatment strictly prefers $q_{0}$ over $q_{1}$, and strictly prefers $q_{i}$ over $q_{i+1}$ for any $i=1, \ldots, n-1$. In addition, if $\theta>0$ is chosen sufficiently high, then a citizen of any type $z \in Z$ prefers to be subjected to the $(\eta, \theta)$-tax treatment than to be a "regular" citizen who pays $c(q)$ and receives no transfer.

Consider the following modifications to the PCSS mechanism: At the selection stage, the proposal $q$ is pitted against $\psi_{-}(q)$ (as before) if $q>\bar{q}$, but is pitted against $\psi_{+}(q)$ if $q<\bar{q}$. If $q=\bar{q}$, then $\bar{q}$ remains in effect. The agenda-setter and the sample group are granted a tax-exemption and receive the transfer of $\mu$ (as before) if the agenda-setter proposes some $q \in Q$ with $q>\bar{q}$, and subsequently $q$ "wins" both at the selection and voting stages. The agenda-setter and the sample group are subjected to the $(\eta, \theta)$-tax treatment if the agenda-setter proposes some $q \in Q$ with $q<\bar{q}$, and subsequently $q$ "wins" both at the selection and voting stages. As before, the special treatment of the sample group imposes a cost on each citizen outside the sample group but this cost vanishes in the limit when the mass of the sample group tends to zero. Indeed, since the PCSS mechanism enhanced with the $(\eta, \theta)$-tax treatment implements the Condorcet winner regardless of the status quo, it has the desirable "dynamic robustness" property.

\section{Discussion, applications, and conclusion}

The main insight of this paper is that democratic decision-making procedures can be used to identify and implement socially desirable policies even in the presence of profound uncertainty. A mechanism solely based on repeated voting does aggregate information truthfully, but is procedurally inefficient. A mechanism based on prior communication among all citizens is prone to strategic behavior. The resolution of profound uncertainty and the implementation of the most socially desirable policy hinge on the use of a conditional privilege for a small sample group as part of the decision-making process.

We stress that the introduced democratic mechanisms are prior-free, that is, they do not depend on the citizens' ex ante beliefs about the states of nature. This is a particularly desirable robustness property of democratic mechanisms, since these mechanisms should 
be applicable to a variety of situations and since their rules should not depend on citizens' current beliefs.

We induce in a small privileged sample of the population a preference for maximizing public good provision which we counterbalance by giving the whole society the power to withdraw the privileges of the sample group by making a minimal downward correction of any over-provision. It is a well-known theme in the political economy literature that sometimes privileged groups such as members of the government bureaucracy would like to maximize public good provision in order to secure their own perks or their "ego rents." One could explore to what extent the corrective present in the selection stage of our PCSS mechanism and the related conditional character of the privileges could be applied to that problem.

There is a variety of extensions and further applications which can be considered in future research. For instance, one could examine to what extent our results carry over to choices from different sets of possible policies, such as continuous policy spaces, or multidimensional public good problems in which several public goods can be combined in a bundle of public goods. Moreover, one might consider an electorate with different income levels and the possibility to differentiate the tax burden as a function of income. In such a model, one could investigate the effect of a policy chosen by a democratic mechanism on the degree of inequality among citizens.

One more relevant question is how the mechanisms discussed in the present paper could be applied in parliamentary settings. Procedural Efficiency is an issue in parliamentary democracy as well since excessively long deliberations on a single issue have opportunity costs, and distract attention from other topics. Procedurally efficient parliamentary decisions might be achieved by a PCSS mechanism with a randomly chosen sample group from the population and a subsequent decision by a majority in parliament.

While such extensions will considerably further the scope of democratic mechanisms in a polity, it is likely that optimal democratic mechanisms with procedural efficiency will involve conditional tax privileges to small groups. In the presence of profound uncertainty we expect such conditional privileges to be an essential ingredient of democratic mechanisms. 


\section{Appendix}

\section{Proof of Lemma 5.4}

Suppose by way of contradiction that there is a Bayesian equilibrium with strategies $\left(\rho^{*}, \sigma^{*}\right)$ which does implement the Condorcet winner on $\mathcal{P}$, while some public good problem $P \in \mathcal{P}$ has the distance property and the state is concealable.

At the communication stage, voting strategy $\sigma^{*}$ assigns a positive or negative message to every type $z \in Z$. The voting strategy $\sigma^{*}$ thus gives rise to the sets

$$
\begin{aligned}
Y_{k}^{\prime} & =\left\{z \in Z \backslash Z_{-} \mid \sigma^{*}(z)=Y e s\right\}, \\
Y_{k}^{\prime \prime} & =\left\{z \in Z_{-} \mid \sigma^{*}(z)=Y e s\right\},
\end{aligned}
$$

of types who vote Yes in state $k$. The measures of the sets $Y_{k}^{\prime}$ and $Y_{k}^{\prime \prime}$ obviously depend on the state. Indeed, for any $k \in N$, let the mass of $Y_{k}^{\prime}$ be $\eta_{k}$ and the mass of $Y_{k}^{\prime \prime}$ be $\chi_{k}$. Moreover, let $\hat{z}=c\left(q_{1}\right) / q_{1}$; that is, all citizens $z<\hat{z}$ belong to $Z_{-}$, while all citizens $z>\hat{z}$ belong to $Z \backslash Z_{-}$. By the supposition that the state is concealable in the public good problem at hand, there is a state $i \in N \backslash\{n\}$ such that the unique maximizer of $d_{i}(z)$ belongs to $Z_{-}$. Let us suppose that $\eta_{i+1} \geq \eta_{i}$. Since $d_{i}$ attains a unique maximum at $z_{i}^{*}<\hat{z}$ and is decreasing on $Z \backslash Z_{-}$, we have that

$$
0 \leq \eta_{i+1}-\eta_{i} \leq F_{i}(\hat{z})-F_{i+1}(\hat{z})
$$

By construction, the set $Z_{-}$has mass $F_{i+1}(\hat{z})$ when the state is $i+1$, hence

$$
0 \leq \chi_{i+1} \leq F_{i+1}(\hat{z})
$$

Adding up the two above inequalities, we obtain

$$
0 \leq \eta_{i+1}-\eta_{i}+\chi_{i+1} \leq F_{i}(\hat{z})
$$

Since $F_{i}(z)$ is a continuous cumulative distribution function, it can attain any value in the interval $\left[0, F_{i}(\hat{z})\right]$ for appropriately chosen $z \in Z_{-}$. In particular, there is a $\bar{z} \in Z_{-}$such that

$$
F_{i}(\bar{z})+\eta_{i}=\eta_{i+1}+\chi_{i+1}
$$

Now consider a joint deviation by the members of $Z_{-}$from $\sigma^{*}$, under which citizens $z \in Z_{-}$with $z<\bar{z}$ send a positive signal, and citizens $z \in Z_{-}$with $z>\bar{z}$ send the negative signal. Under this deviation, the share of positive signals among all citizens in state $i$ is equal to the share of positive signals under voting strategy $\sigma^{*}$ in state $i+1$. Recall the supposition that the Condorcet winner is 
implemented under $\left(\rho^{*}, \sigma^{*}\right)$. This supposition implies that the proposal strategy $\rho^{*}$ has agendasetters of all types propose $q_{i}$ if they observe the share $\eta_{i}+\chi_{i}$ of positive signals. Therefore, if the share $F_{i}(\bar{z})+\eta_{i}=\eta_{i+1}+\chi_{i+1}$ of positive signals is observed, then with probability one, the proposal in the decisive voting round will be $q_{i+1}$. Thus, under the deviation considered here, the proposal $q_{i+1}$ will be made at the voting stage with probability one if the true state is $i$. Since citizens vote sincerely, this proposal will be rejected, and the resulting public good level will be $q_{0}$. By construction, members of $Z_{-}$prefer $q_{0}$ over $q_{i}$. Now we have shown that the deviation by $Z_{-}$ is (strictly) profitable if the true state is $i$. Suppose next that the true state is some $j \in N \backslash\{i\}$. In that state, no quantity $q \in Q$ with $q>q_{j}$ would be accepted at the voting stage. In the supposed Nash equilibrium, the outcome is $q_{j}$. Thus, under the deviation, the outcome must be some $q_{j}^{\prime} \leq q_{j}$ if the state is $j$. All members of $Z_{-}$weakly prefer $q_{j}^{\prime}$ to $q_{j}$. Since $p_{i}>0$, the deviation is (strictly) profitable. It follows that in the supposed equilibrium, the state cannot be revealed through this mechanism. ${ }^{16}$

We have now completed the proof of the lemma for the case where $\eta_{i+1} \geq \eta_{i}$. We can repeat the argument by alternatively defining $\eta_{k}$ and $\chi_{k}$ as the share of negative rather than positive signals in state $k \in N \backslash\{n\}$, and by constructing a joint deviation for members of $Z_{-}$such that citizens $z<\bar{z}$ send the negative signal, and citizens $z \in Z_{-}$with $z>\bar{z}$ send the positive signal. In this sense, the earlier supposition that $\eta_{i+1} \geq \eta_{i}$ is without loss of generality, and thus the proof of the lemma is complete.

\section{Proof of Theorem 7.1}

Consistent beliefs. It is straightforward that the belief $\pi^{\bar{z}}$ is consistent with the signaling strategy $\sigma^{\bar{z}}$. Moreover, on the path of play induced by $\left(\pi^{\bar{z}}, \rho^{\bar{z}}, \sigma^{\bar{z}}\right)$, the agenda-setter proposes the Condorcet winner, and thus the belief $\pi^{*}$ is consistent.

Implementation. Given beliefs $\pi^{*}$ and $\pi^{\bar{z}}$, all citizens believe that both the proposal made by the agenda-setter and the amendment would prevail against the status quo at the voting stage. Thus, $\left(\bar{\omega}_{s}^{*}, \bar{\omega}_{p}^{*}\right)$ prescribes sincere selection by citizens outside the sample group and voting for the

\footnotetext{
${ }^{16}$ We have an equilibrium concept which allows types with the same preference ranking over $Q$ to coordinate deviations. The reasoning in the proof at hand is based on a joint deviation by the members of $Z_{-}$who coordinate to mislead the agenda-setter. One could argue that the agenda-setter should be assumed to be aware of the deviation by $Z_{-}$if the agenda-setter himself belongs to that group. Then, an agenda-setter $z \in Z_{-}$would know the true state and propose the "right" Condorcet winner $q_{i}$ despite observing the "wrong" share $\delta_{i+1}$ of positive votes. However, this happens with a probability strictly less than one since $Z \backslash Z_{-}$has strictly positive measure. Thus, the argument remains valid: The members of $Z_{-}$expect to gain from the joint deviation which we have constructed.
} 
proposal for the sample group members. Since the proposal is the Condorcet winner on the path of play, a share of at least $(1+\lambda) / 2$ select the proposal. Because of sincere voting at the voting stage and the fact that the proposal is the Condorcet winner, the Condorcet winner does become the final outcome of the mechanism, as desired.

Optimal selection. We have constructed $\left(\bar{\omega}_{s}^{*}, \bar{\omega}_{p}^{*}\right)$ to be optimal.

Optimal signaling and agenda-setting. Now we show that no deviation by the agenda-setter or by sample group members can be profitable. Notice that sample group members and agenda-setter receive the payoff $z q_{i}+\mu$ on the path induced by the profile under consideration when the state is $i$. For every $i \in N$, let $\hat{q}_{i}$ be the outcome which follows after a profitable deviation. Since the deviation is supposed to be profitable, there must be a state $i \in N$ such that $\hat{q}_{i}>q_{i}$. Suppose indeed that such a state $i$ occurs. Due to the sincere selection strategy, any proposal $q \in Q$ such that $q>q_{i}$ will not be selected. In particular, $\hat{q}_{i}$ will not be selected. Thus the outcome $\hat{q}_{i}>q_{i}$ must have been an amendment at the selection stage, and must then have prevailed against $q_{0}$ at the voting stage. Hence, under the deviation, there is no tax exemption and no transfer. Since the deviation is profitable for an agenda-setter or sample group member of type $z$, it must hold that $z \hat{q}_{i}-c\left(\hat{q}_{i}\right)>z q_{i}+\mu$. However, for any $z \in Z$, this inequality is violated for sufficiently large $\mu$. Thus, we obtain a contradiction for large enough $\mu$. 


\section{References}

Aghion, P. and P. Bolton (2003) "Incomplete social contracts," Journal of the European Economic Association, 1, 38-67.

Bednar, J. (2011) "Nudging federalism towards productive experimentation," Regional and Federal Studies, 4-5, 503-521.

Bendor, J. AND D.MoOKherJeE (1987) "Institutional structure and the logic of ongoing collective action," American Political Science Review, 81, 129-154.

Bergemann, D. And S.Morris (2005) "Robust mechanism design," Econometrica, 73, 1771-1813.

Bernhardt, D., J. Duggan and F.Squintani (2008) "A survey on polling in elections," in Aragones, E., Bevia, C., Llavador, H., Schofield, N., Eds., The Political Economy of Democracy, Fundacion BBVA.

Britz, V. and H.Gersbach (2014) "Experimentation in Democratic Mechanisms," Center of Economic Research at ETH Zurich Working Paper No. 14/199.

Buchanan, J.M. and G.Tullock (1962) "The Calculus of Consent: Logical Foundations of Constitutional Democracy." Ann Arbor: University of Michigan Press.

Buera, F.J., A.Monge-Naranjo, And G.E.Primiceri (2011) "Learning the wealth of nations," Econometrica, 79, 1-45.

CaI, H. And D.Treisman (2009) "Political decentralization and policy experimentation," Quarterly Journal of Political Science, 4(1), 35-58.

Callander, S. And B.Harstad (2013) "Experimentation in federal systems," NBER Working Paper 19601.

Crawford, V.P. And J.Sobel (1982) "Strategic information transmission," Econometrica, 50, 14311451.

Gersbach, H. (2009) "Democratic mechanisms," Journal of the European Economic Association, 7, 1436-1469.

Gersbach, H., Hahn, V., And S.Imhof (2013) "Tax rules," Social Choice and Welfare, 41, 19-42.

Kollman, K. (2003) "The rotating presidency of the European Council as a search for good policies," European Union Politics, 4, 51-74.

Kollman, K., J.H.Miller, and S.E.Page (2000) "Decentralization and the search for policy solutions," Journal of Law, Economics, and Organization, 16, 102-128.

Meirowitz, A. (2003) "Polling games and information revelation in the Downsian framework," Games and Economic Behavior, 51, 464-489.

Morgan, J. And P.C. Stocken (2008) , "Information aggregation in polls," American Economic Review, 98, 864-896.

Rose-Ackerman, S. (1980) "Risk-taking and reflection: Does federalism promote innovations?" 
Shipan, C.R. And C.Volden (2006) "Bottom-up federalism: The diffusion of anti-smoking policies from U.S. cities to states," American Journal of Political Science, 50, 825-843.

StrumpF, K.S. (2002) "Does government decentralization increase policy innovation?" Journal of Public Economic Theory, 4, 207-241.

Volden, C. (2006) "States as policy laboratories: Emulating success in the children's health insurance program," American Journal of Political Science, 50, 294-312.

Volden, C., M.M.Ting, And D.P.CArPenter (2008) "A formal model of learning and policy diffusion," American Political Science Review, 102, 319-332. 